\title{
Integrating Scientific Publication into an Applied Gaming Ecosystem
}

\author{
Munir Salman \\ FernUniversität in Hagen \\ Faculty for Multimedia and \\ Computer Science \\ Hagen, Germany \\ Email: munir.salman@ \\ studium.fernuni-hagen.de
}

\author{
Michael Fuchs \\ Wilhelm Buechner \\ University of Applied \\ Science \\ Pfungstadt, Germany \\ Email:mfuchs@ftk.de
}

\author{
Binh Vu, Holger Brocks, \\ Jana Becker \\ Research Institute for \\ Telecommunication \\ And Cooperation \\ Dortmund, Germany \\ Email: Bvu@ftk.de \\ Email: hbrocks@ftk.de \\ Email: jbecker@ftk.de
}

\author{
Dominic Heutelbeck \\ Research Institute for \\ Telecommunication and \\ Cooperation \\ Dortmund, Germany \\ Email: dheutelbeck@ftk.de
}

\begin{abstract}
The European (EU)-based industry for non-leisure games (so called Applied Games, AGs) is an emerging business. As such it is still fragmented and needs to achieve critical mass to compete globally. Nevertheless, its growth potential is widely recognized and even suggested to exceed the growth potential of the leisure games market. The European project Realizing an Applied Gaming Ecosystem (RAGE) is aiming at supporting this challenge. RAGE will help to seize these opportunities by making available an interoperable set of advanced Applied Game (AG) technology assets, as well as proven practices of using such AG assets in various real-world contexts. As described in [1], RAGE will finally provide a centralized access to a wide range of applied gaming software modules, relevant information, knowledge and community services, and related scientific documents, taxonomies, media, and educational resources within an online community portal called the RAGE Ecosystem. Besides this, an integration between the RAGE Ecosystem and relevant social network interaction spaces that arranges and facilitates collaboration that underlie Research and Development $(R \& D)$, as well as marketoriented innovation and exploitation will be created in order to support community building, as well as collaborative asset exploitation of User Generated Contents (UGCs) of the RAGE Ecosystem. In this paper, we will describe the integration of the Scientific Publication Platform (SPP) Mendeley [2] into the RAGE Ecosystem. This will allow for automating repetitive tasks, reducing errors, and speeding up time consuming tasks. On the other hand it will support information, UGC, and knowledge sharing, as well as persistency of social interaction threads within Social Networking Sites (SNSs) and Groupware Systems (GWSs) that are connected to the RAGE Ecosystem. The paper reviews relevant use cases and scenarios, as well as related authentication, access, and information integration challenges. In this way, on the one hand a qualitative evaluation regarding an optimal technical integration is facilitated while on the other hand design approaches for supporting features of resulting user interfaces are initiated.
\end{abstract}

Keywords-Social Networking; Applied Gaming; Digital Ecosystem; Access and Information Integration; Know-how transfer; scientific publication; Mendeley

\section{INTRODUCTION AND MOTIVATION}

The EU-based industry for Applied Games (AGs) is an emerging business. As such it is still fragmented and needs to achieve critical mass to compete globally. Nevertheless, its growth potential is widely recognized and even suggested to exceed the growth potential of the leisure games market. The RAGE project [3] is aiming at supporting this challenge. RAGE will help to seize these opportunities by making available an interoperable set of advanced technology assets, tuned to applied gaming, as well as proven practices of using asset-based applied games in various real-world contexts. This will be achieved by enabling a centralized access to a wide range of applied gaming software modules, information, knowledge and community services, as well as related document, publication, media, and educational resources within the RAGE Ecosystem. Furthermore, the RAGE project aims to boost the collaboration of diverse actors in the AG environment. Therefore, the main objectives of the RAGE Ecosystem are to allow its participants to get hold of advanced, usable gaming assets (technology push), to get access to the associated business cases (commercial opportunity), to create bonds with peers, suppliers, and customers (alliance formation), to advocate their expertise and demands (publicity), to develop and publish their own assets (trade), and to contribute to creating a joint agenda and road-map (harmonization and focus).

This means that seen as a whole, the RAGE project is a technology and know-how driven research and innovation project. Its main driver is to be able to equip industry players (e.g., game developers) with a set of AG technology resources (so-called Assets) and strategies (i.e., know-how being provided by means of information services and knowledge resources) to strengthen their capacities to penetrate a market (non-leisure), which is new for most of them, and to consolidate a competitive position in it. Fig. 1 represents the positioning of the project in the spectrum from 'theory to application'.

In consequence, the RAGE Ecosystem and its integration with social networks of game-research-, game-developing- , gaming-, and AG communities will on the one hand become an enabler to harvest community knowledge and on the other hand it will support the access of such communities to the RAGE Ecosystem as an information and knowledge resource.

Building on the results of the Social Networking Sites (SNSs) and Groupware Systems (GWSs) integration with the

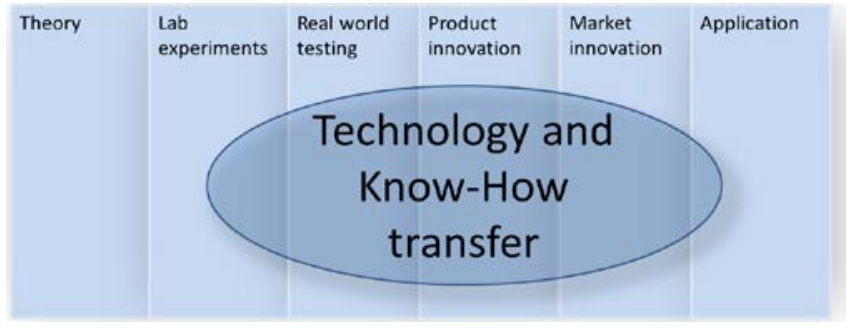

Figure 1. Technology and Know-How transfer [1] 
RAGE Ecosystem including corresponding SNS-enabled content and knowledge management, the RAGE Ecosystem will in the future also support Social Network Analysis (SNA) by means of applying technologies for Natural Language Analysis (NLA) for discourse analysis, as well as Named Entity Recognition (NER) and Semantic Representation and Annotation (SRA) of its results [4]. This will, e.g., enable users to utilize the envisioned Ecosystem with features of a social mediation engine going beyond content syndication, i.e., it will serve as a social space that mediates collaboration partners, while content remains the main attractor. Finally, an interactive map of supply- and demand-side stakeholders and resources will be provided for domain and community orientation, as well as visual access support.

In the remainder of this paper, section II provides a brief introduction of a set of exemplar target communities that are present in SNSs and GWSs. Furthermore, section III describes related research activities of these communities. Section IV is about state of the art in science and technology. Section V, more specifically, reviews the integration possibility of Mendeley and its Application Programming Interface (API) that supports integration with the RAGE Ecosystem. Furthermore, this section will investigate how to support a bi-directional access to resources, assets and community information between the RAGE Ecosystem and such SNSs and GWSs. Next, section VI will outline design approaches towards supporting users in the target communities by services provided by the RAGE Ecosystem by means of outlining several use case scenarios for using Social Networking Features (SNF) and Groupware Features (GWF) within the RAGE Ecosystem user interfaces. Finally, the paper will present conclusions and future work.

\section{TARGET SNS AND GWS USER COMMUNITIES AND CORRESPONDING EXEMPLAR USER STEREOTYPES}

As outlined above, the EU-based industry for AG is an emerging business, which is still fragmented and needs to achieve critical mass for global competition. The AG industry and developer groups want to keep their developments innovative, i.e., attractive and technologically in good condition. These groups already have a very good understanding of their competitive advantage and corresponding assets (e.g., software, documents, and social media objects, etc.). However, they also need innovative ideas to develop innovative AGs in order to stay competitive. Therefore, they look for possibilities to cooperate with AG Research and Development (R\&D) groups. Besides this, the AGs that researchers create within research projects produce a lot of AG research assets and prototypes, which need to be fully developed and deployed by AG software developers to become marketable. Apart from AG developers and researchers, there are also AG customers and players who on the one hand want to learn about or contract the development of AGs and on the other hand can also contribute to the development of AG usage scenarios. Many of these communities (AG developers, researchers, customers and players) are already present in a fragmented way within several groups in several SNSs and GWSs. In [1] we have presented some examples of AG research, as well as industry and developer communities in, e.g., LinkedIn and Twitter. The Applied Games and Gamification (AGG) LinkedIn group [5] has over 4,500 members and has been running since 2011. The group claims to be one of the largest collective of creators, developers, researchers, and users of applied games and gamification globally. The typical users can be distinguished roughly into those from industry and those from academia, i.e., from professors and recent graduates in gaming and related technologies, to CEOs, founders and directors of a wide variety of organisation that work or research the domain. The majority of discussion posts are promotions of products, methodologies for design, reposts of other interesting blogs on the topic and individuals' thoughts on implications of games and gamification for learning, training and behaviour change. The most prolific posters tend to be consultants and individuals representing organisation that are looking to showcase their abilities to a more business oriented community toward winning more business. Many posts do not garner comments or discussion as they are often pointing to other resources; however posts which pose interesting questions do receive attention and lead to interesting discussions from the more active members. Similarly the Serious Games Group (SGG) on LinkedIn [6] has over 5200 members and has been running since 2008. Another AG research group example is the Game Research (GR) Mendeley group which has more than 140 members and more than 200 papers. The group memberships somewhat overlap with the applied games and gamification, however the audience tends to be more focused on the learning solutions and learning providers, with fewer CEOs and marketing directors, and more game designers as compared to the AGG, although the mode of use are very similar.

RAGE will help to overcome this fragmentation and aims to support the capturing, as well as the representation, management, sharing, and exchange of social media produced content and knowledge resources through its Ecosystem. Therefore, the integration of SNSs and GWSs hosting such target communities with the RAGE-Ecosystem and at the same time enabling the connectivity between SNSs, GWSs, and the RAGE-Ecosystem will connect research-, gaming industry-, intermediary-, education provider-, policy maker- and end-user communities. Furthermore, it will facilitate the centralized access to the valuable assets beyond the SNSs and GWSs.

As a whole take-up of RAGE results will generate impacts that will be visible through multiple enhancements in the performance of European Applied Game industries, especially in terms of reducing the current fragmentation, improving their innovation capacity and fostering their progress towards global technological leadership. By offering reusable Applied Games assets, the RAGE Ecosystem infrastructure and marketplace will play a key role in support of applied research and technology development, including demand driven research and productification activities, easing technology transfer and field validation of novel products and services, on a broad 
collaborative basis. The combined effects will allow end-toend Applied Games value chain players to dramatically improve their competitive position.

\section{RELATEDWORK}

The work presented in this paper is related to a number of topics in research. The RAGE Ecosystem will be built upon the Educational Portal (EP) technology and application solution, which was developed by the software company GLOBIT [7] that already was used in the Alliance Permanent Access to the Records of Science in Europe Network (APARSEN) [8]. APARSEN was an EU-funded project within the digital preservation area with the goal to create a virtual research center in digital preservation in Europe. The so-called EP tool-suite offers a wide variety of tools and is currently extended by Research Institute for Telecommunication and Cooperation (FTK) within the RAGE project into an Ecosystem Portal (EP) tool suite [9]. This includes a web based, user-friendly User and Community Management (UCM) including an advanced Contact and Role Management (CRM) based on MythCRM [10], as well as knowledge management support in the form of Taxonomy Management (TM) support and semiautomatic taxonomy-based Content Classification (CC) support [4], [11], as well as a Learning Management System (LMS) based on Moodle [12] and an advanced Course Authoring Tool (CAT) [13]. In this way, the Content \& Knowledge Management (C\&KM) tools of the EP tool suite support the management of documents in a taxonomysupported Digital Library, the management of multimedia objects in a taxonomy-supported Media Archive and the management of Learning Objects in a competence-based LMS [14]. Furthermore, one of its additional purposes is to support Continuous Professional Education (CPE) and training of practitioners, experts, and scientists, which are members of professional communities of practice or scientific communities. Fig. 2 displays the components and services in the EP tool suite as described in [4]. EP was built based on Typo3 [7] and, therefore, can be extended with the help of Typo3 extensions. Evgeny, Bogdanov et al [15] extend a social media platform in higher education with lightweight tools (widgets) aimed for collaborative learning and competence development. Our work will establish the new EP module Community \& Social Network Support (CSNS) on the basis of a so-called Agile Application Programming Interface (AAPI), which facilitates the connectivity to a wide range of SNSs and GWSs.

\section{RELEVANT STARTING POINTS WITHIN THE STATE OF THE ART IN SCIENCE AND TECHNOLOGY}

SNSs and GWSs have changed the way of information sharing and learning processes by adding innovative features to social communication. SNSs were defined as "Internet or mobile-device based social spaces designed to facilitate communication, collaboration, and content sharing across networks of contacts. SNS allows its users to become content creators and content consumers at the same time, thus allowing instant participation, sharing of thoughts or information and personalised communication" [16]. Therefore, SNSs and GWSs are becoming increasingly important. This holds especially true for various Social

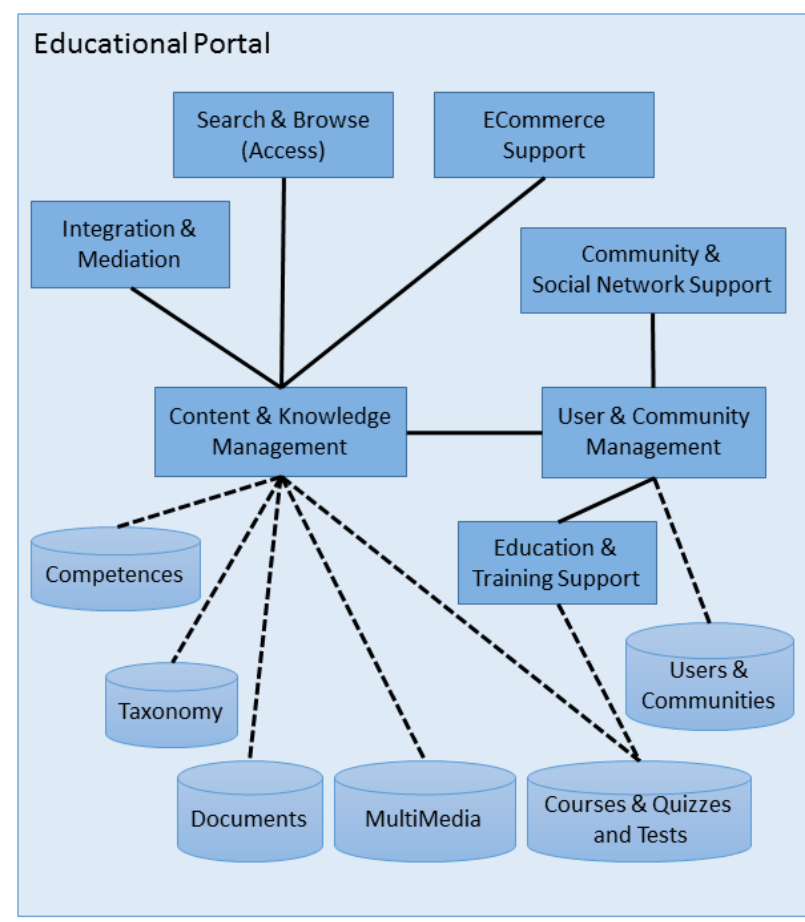

Figure 2. EP Tool Suite - Components and Services

Networking Features (SNFs) and Groupware Features (GWFs) like, e.g., rating, commenting, tagging, chatting, liking, posting new Social Media (SM) and User Generated Content (UGC), following actors or celebrities, playing games etc. These SNFs and GWFs are not only entertaining and exciting but also useful for learning and for information enrichment. Research has shown that distance education courses are often more successful when they develop communities of practice [17]. Besides, SM content becomes increasingly important in business and research. Kaplan [18] gave a clarification what the term SM means and how the concept of SM differs from the concept of related concepts such as Web 2.0 and UGC. Furthermore, he presents 10 pieces of advice to utilize SM. On the other hand, Agichtein et al. [19] focus on the Semantic Social Media (SSM) and investigate methods exploiting community feedback, e.g., to automatically identify high quality content. Breslin et al. define in [20] "The Social Semantic Web as a vision of a Web where all of the different collaborative systems and social network services, are connected together through the addition of semantics, allowing people to traverse across these different types of systems, reusing and porting their data between systems as required." RAGE will use Semantic Web technologies in order to describe in an interoperable way users' profiles, social connections, and social media creation and sharing across different SNSs and GWSs, as well as within the RAGE Ecosystem. Therefore, RAGE will be able to deliver well-grounded recommendation and mediation features AG R\&D communities.

Today, most SNSs and GWSs provide so-called Application Programming Interfaces (APIs) for developers to integrate the SNSs and GWSs into their systems. Although, the SNSs and GWSs are different in their functionality, i.e., their social networking feature support, their software architecture 
for the communication with distributed other systems is similar. Most of the SNSs and GWSs offer REST APIs like [2], [21], [22] which can be used for integration with other systems.

In the following, the description of the Mendeley API software architecture as described in [2], [23] will be cited as an exemplary, illustrative, and at the same time representative example.

In summary, it is a big advantage to aim at supporting the integration of SNSs and GWSs including relevant SNFs and GWFs, as well as UGC capturing, management, sharing, and dissemination support through their REST API into the RAGE Ecosystem. This will on the one hand facilitate to extend the envisioned RAGE Ecosystem with features of a social mediation engine going beyond content syndication, i.e., it can serve a social space that mediates collaboration partners, while content remains the main attractor. On the other hand it focuses on identifying collaboration opportunities between individuals and among groups, to support matchmaking and collaboration between stakeholders, and to identify and provide support for innovation opportunities and creativity efforts. That allows communities (such as technology providers, game developers and educators, game industries, researchers) to create their own assets and post them to the Ecosystem's repository without major effort. Besides this, the above approach enables follow-up work in the area of social network analysis and discourse analysis, which can then be conducted and used to provide feedback, recommendations, mediations, and relevant information to the communities. This feedback can e.g., help gaming companies to develop new markets in applied gaming.

\section{INTEGRATION APPROACH AND IMPLEMENTATION}

The following section presents the main technical integration possibilities in the backend, as well as in frontend. In this way, our integration approach and methodology is enabling us to differentiate between how to get access to resources and assets in the RAGE Ecosystem from external SNSs and GWSs communities and how to push contents from the RAGE Ecosystem to the external SNSs and GWSs in order to improve user acceptance of services provided by the RAGE Ecosystem. Fig. 3 displays the concept of a bi-directional integration approach of the RAGE Ecosystem with SNSs (e.g. LinkedIn

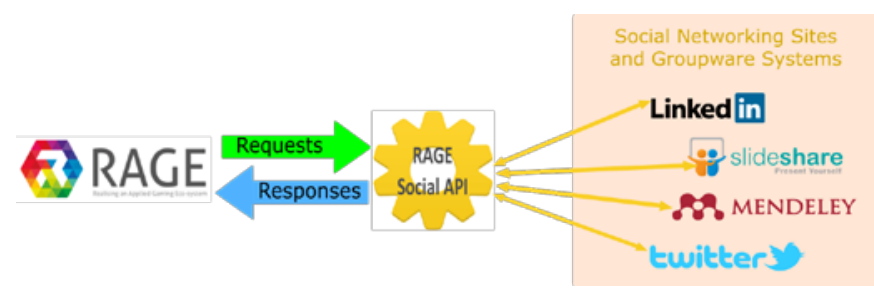

Figure 3. Integration Approach of RAGE Ecosystem with SNSs and GWSs

and Twitter) and GWS (e.g. Mendeley and GitHub) using a
REST API. Corresponding to this bi-directional integration approach, the Tight and Loose Coupling methodologies, as described in [24], will be considered for achieving an integration of SNSs and GWSs to the RAGE Ecosystem and vice versa.

As an example, the so-called Mendeley API and its software architecture as described in [2] will be cited as an exemplary, illustrative, and at the same time representative example for the loose and tight coupling between the RAGE Ecosystem and GWS.

The Mendeley API is based upon the following standard:

- RESTful using the base URL: https: //api . mendeley.com

- JSON: The response Data is delivered in JSON format.

- HTTPS: All requests must be send using TLS/SSL connections.

- OAuth 2.0 is used to authenticate and authorize all Mendeley API requests.

- CORS: Cross original resource sharing is enabled on all API requests. CORS is a mechanism to enable client-side cross-origin requests [25].

Each Mendeley's resource is identified by a URL path. There are many types of data available but the principal resources include [2]:

- /document: User Documents created by Mendeley users and assigned to either Libraries or Groups

- /catalog: Catalog Documents from Mendeley’s crowdsourced collection of papers

- /files: File attachments associated with User Documents

- /groups: Collaborative users form a group for sharing documents and ideas

- /annotations: Users mark up and comment in documents and files using annotations

Furthermore, Mendeley provides a Software Development Kit $(S D K)$ to ease client application development. Using the Mendeley JavaScript SDK [23] facilitates the tight coupling integration of the Mendeley within the RAGE Ecosystem. This SDK includes code for the implicit grant and authorization code flows. For the RAGE Ecosystem we used the authorization code flow in order to acquire an access token. The RAGE server will do the token exchange and set the access token cookie. From the client-side point of view the flow could be started as shown in the following code.

Corresponding to this authorization code flow, Fig. 4 displays the successful login user interface through the RAGE Portal.

The Mendeley JavaScript SDK is available as an var options $=\{$ apiAuthenticateUrl: '/login', refreshAccessTokenUrl: '/refreshtoken'

\} ;

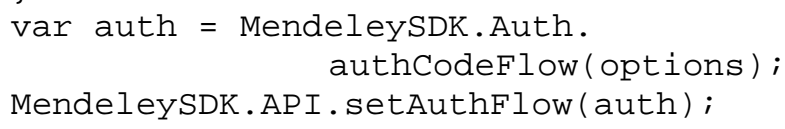




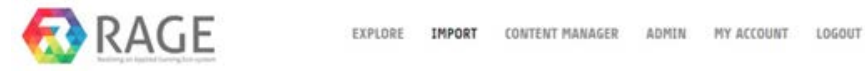

Import from Mendeley

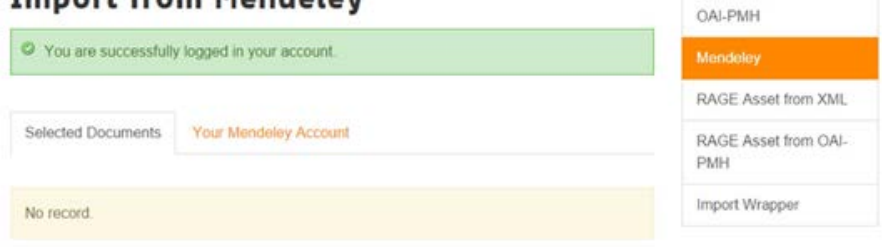

Figure 4. Successful login User Interface

Asynchronous Module Definition (AMD) or a standalone library. The following code shows an example using the standalone library for capturing data for the user after the authentication is completed. Each call will either resolve with some data or reject with the original request and the API response [23].

MendeleySDK. API . documents . list ( ) . done (fu nction(docs) \{ console. $\log ($ 'Success!' );

console. $\log ($ docs $)$;

\}). fail(function(request, response) \{ console. $\log$ ( 'Failed!' );

console. $\log ($ 'URL:', request.url); console. $\log$ ('Status:',

response.status );

\});

Fig. 5 is the interface of above code, which shows the import support for documents from Mendeley into the RAGE Ecosystem of the logged in user.

\section{VI.SNF USAGE SCENARIOS AND DESIGN CONCEPT}

In addition to outlining our SNS integration approach and methodology, Fig. 6 displays how the SNS usage scenarios can be integrated into the RAGE Ecosystem itself. RAGE Ecosystem users can visit

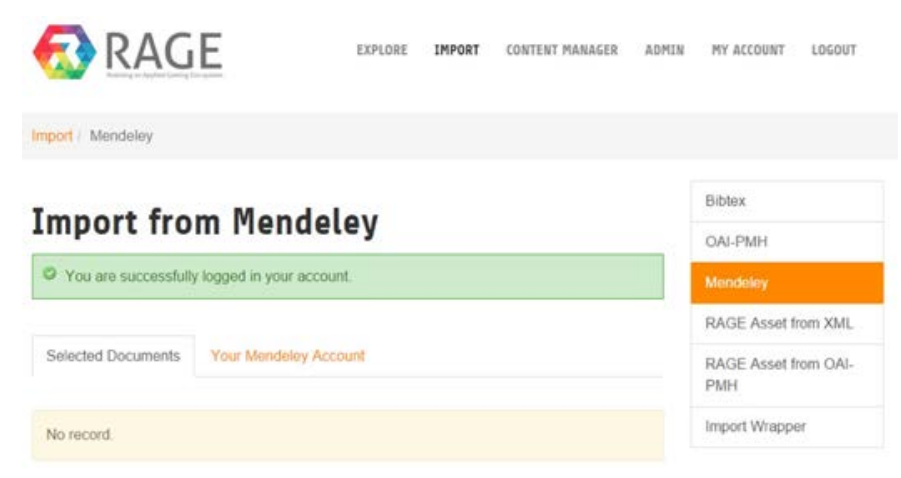

Figure 5. Importing documents from Mendeley
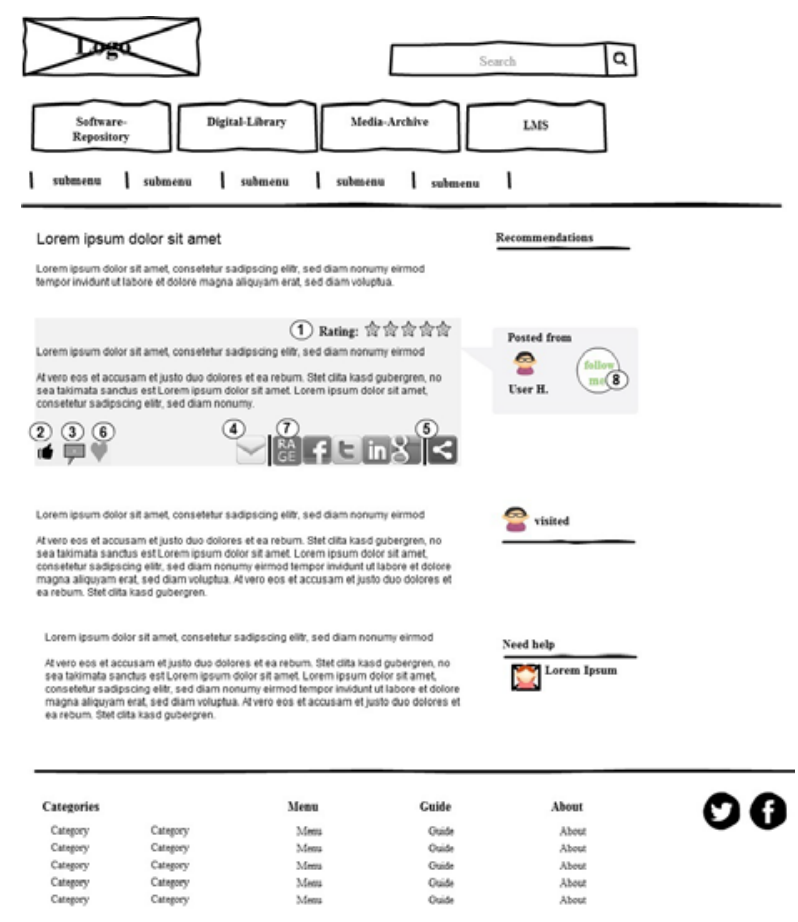

Figure 6. SNF Usage Scenario in the RAGE Ecosystem

content and knowledge management support within the RAGE Ecosystem's a Digital Library, Media Archive, Software Repository (which is currently under development based on [17]), and LMS. Here, users have the opportunity to:

a. Rate (1), like (2), and Comment (3): these Social Networking Features (SNFs) are e.g., important for the recommendation system (also currently under development [16] to get more useful suggestions.

b. Tell a friend (4): users can send links to selected content (or the content itself) through email. Email addresses can be selected either from the RAGE address book or from users' address books, which are located in SNSs.

c. Share and post (5): Users can share the selected content to one of their favourite SNSs or on the fly to more than one by selecting them from the share button.

Users also have the possibility to publish content to a repository (e.g., GitHub's repository) or to cloud storage (e.g., into Dropbox).

d. Favourite (6): Users can add content to their favourite lists, which facilitates to later, e.g., share/post their entire favourite list to a community.

e. Share and post to RAGE Communities (7) within the RAGE Ecosystem and also from any other platforms outside the RAGE Ecosystem. A RAGE Share-Button can be released and, e.g., be integrated by developers 
into other portals, homepages, ecosystems etc. to provide the possibility to Internet Users to share and post their content to the RAGEEcosystem.

f. RAGE Follow-me (8): RAGE users can follow other users, groups or content in order to keep themselves up-to-date.

\section{CONCLUSION AND OUTLOOK}

In this paper, we have introduced the RAGE Ecosystem supporting community-based content and knowledge management. In detail it will support the collection, sharing, access, and re-use of AG R\&D assets, including UGC resources, as well as academic, industry, and end user best practice knowhow represented in corresponding knowledge resources. In this way, the RAGE Ecosystem will provide AG communities, and therefore SNSs and GWSs communities, too, an opportunity to interact, share and re-use content and UCG including corresponding knowledge resources, as well as communicate and collaborate using the RAGE Ecosystem as a back-end community content and knowledge management portal in addition to their favorite SNSs and GWSs. Besides this introduction, we have presented how a technical integration between the RAGE Ecosystem and SNSs and GWSs can be achieved to reduce the fragmentation and to increase the knowledge exchange among AG communities (such as AG developers, researchers, customers, and players). The RAGE Ecosystem and its SNSs, SNFs, GWSs and GWFs integration are currently under development. In the future, RAGE is aiming at increasing outreach and take-up of the RAGE Ecosystem through further SNSs and GWSs integration and SNFs and GWFs implementation. For example, the SNA and discourse analysis will be used for collecting, analyzing, and presenting data about various patterns of relationships among people, objects, and knowledge flows within the RAGE Ecosystem and will provide additional functionality and sophisticated services for end-users, enhancing the emergence of communities. In particular, future developments will focus on identifying collaboration opportunities among individuals and groups, to support matchmaking and collaboration among main stakeholders, and to identify and provide support for innovation opportunities and creativity efforts. In this way, the RAGE project currently anticipates the following tools and services:

a) The RAGE Diagnostic tool based on various metrics for analyzing the usage of resources, the formation of different users groups, the level of social interactions, etc.,

b) the RAGE awareness tool can increase participation of different target groups in the Ecosystem,

c) the RAGE Knowledge Mapping tool builds and analyses knowledge maps for all kind of resources available in the Ecosystem.

d) the RAGE Professional support tool will support the users by letting them know whom or where to ask for support in different situations,

e) the RAGE Community detection tool will use available clustering algorithms (also called “community detection algorithms") that automatically identify and locate existing communities, in order to enhance the communication between gaming practitioners,

f) the RAGE Ecosystem analysis tool will apply network analysis including many algorithms for identifying the most important, or central in some sense, nodes within a network,

g) the RAGE Recommendation may generate value interventions towards stimulating the participation of users. Such interventions include suggesting connections among users, setting up groups, closing the gaps in people's knowledge of other members' expertise and experience, and strengthening the cohesiveness within existing teams. Social media data such as tags, comments, purchasing patterns, and ratings can be used to link related gaming assets and users together into networks, the RAGE Social learning tool applies SNA to online learning environments, as well, focusing on the structural relationships between all learning objects and users, that support learning communities.

With the design and development of a comprehensive approach as pursued with the RAGE Ecosystem, ethical issues need to be taken into account. The integration of users' SN profiles from different SNSs, as well as the use of features carrying out analyses on top of Ecosystem user data have ethical implications in terms of privacy and data protection and require appropriate information and consent in the terms and conditions of use, as well as compliance to national and international data protection regulations. The same is for any use of log data for the purpose of system evaluation, or for UGC and user actions shared among different SNSs and the RAGE Ecosystem. In addition, with UGC questions related to verification and validation of contributions, as well as to copyright ownership and infringement become relevant. The consideration of such ethical and legal requirements shall be incorporated in the system design and development process in terms of an ethics-by-design approach [26]. This means that data protection and privacy is already taken into account when the system is being designed. Design principles, such as purpose binding, would ensure that personal information is only accessible, if there is a need for it when performing a certain action. The system can also control data access by respecting personal settings which data should be available to others or the public. Other ethics-enabled features include the modification or deletion of personal data.

\section{ACKNOWLEDGEMENTS AND DISCLAIMER}

This publication has been produced

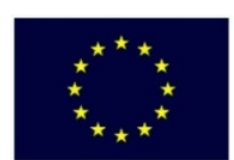
in the context of the RAGE project. The project has received funding from the European Union's Horizon 2020 research and innovation programme under grant agreement No 644187. However, this paper reflects only the author's view and the European Commission is not responsible for any use that may be made of the information it contains. 


\section{REFERENCES}

[1] M. Salman, D. Heutelbeck, and M. Hemmje, 'Towards Social Network Support for an Applied Gaming Ecosystem', in Proceedings of the 9th European Conference on Games Based Learning ECGBL 2015, Steinkjer, Norway, 2015, pp. $721-728$.

[2] 'Mendeley Developer Portal'. [Online]. Available: http://dev.mendeley.com/. [Accessed: 04-Nov-2015].

[3] netvision, 'RAGE', RAGE. [Online]. Available: http://rageproject.eu/. [Accessed: 05-Nov-2015].

[4] C. Nawroth, M. Schmedding, H. Brocks, M. Kaufmann, M. Fuchs, and M. Hemmje, 'Towards Cloud-Based Knowledge Capturing Based on Natural Language Processing', in Procedia Computer Science, 2015, vol. 68, pp. 206-216.

[5] 'Applied Games and Gamification | LinkedIn'. [Online]. Available: https://www.linkedin.com/grps/AppliedGames-Gamification-3889283/about? [Accessed: 25Nov-2015].

[6] 'Serious Games Group | LinkedIn'. [Online]. Available: https://www.linkedin.com/grps/Serious-GamesGroup-137156/about? [Accessed: 25-Nov-2015].

[7] 'Globit'. [Online]. Available: http://globit.com/. [Accessed: 20-Nov-2015].

[8] S. Schrimpf, 'APARSEN - Alliance Permanent Access to the Records of Science in Europe Network.', pp. 52-53, Feb. 2014.

[9] D. Binh Vu, 'Realizing an Applied Gaming Ecosystem Extending an Education Portal Suite towards an Ecosystem Portal', Master Thesis, Technische Universität Darmstadt, Darmstadt, Germany, 2015.

[10] D. Binh Vu, 'Customer Relationship Management based on Identity Management for Scientific Associations', Bachelor Thesis, Technische Universität Darmstadt, Darmstadt, Germany, 2015.

[11] T. Swoboda, 'Towards effectivity augmentation of automated scientific document classification by continuous feedback', Masterthesis, Fernuniversität Hagen, Hagen, 2014.

[12] 'Lernerfolg mit Moodle'. [Online]. Available: http://moodle.de/. [Accessed: 25-Nov-2015].

[13] Zentrum fuer Medien und IT (ZMI), M. Then, B. Wallenborn, B. R. Ianniello, M. Hemmje, FernUniversitaet in Hagen, and Lehrgebiet Multimedia und Internetanwendungen, 'Learning Design and Integration of Legacy Tools into Modern Learning Platforms Towards supporting Competence-Based Course Concepts within E-Learning Platforms and Legacy Assignment Tools by Means of Innovative Authoring Tools.', 2015.

[14] R. Koper, 'TENCompetence: building the European network for lifelong competence development'.
[15] E. Bogdanov, F. Limpens, N. Li, S. El Helou, C. Salzmann, and D. Gillet, 'A social media platform in higher education', in 2012 IEEE Global Engineering Education Conference (EDUCON), 2012, pp. $1-8$.

[16] I. Journals and J. M. R. S. 2,Dr. K. Puttaraju , 1, 'A Study on Gender Differential Factors in Uses of Social Networking Sites’. 28-Jan-2015.

[17] S. A. Barab and T. M. Duffy, From Practice Fields to Communities of Practice. .

[18] A. M. Kaplan and M. Haenlein, 'Users of the world, unite! The challenges and opportunities of Social Media', Bus. Horiz., vol. 53, no. 1, pp. 59-68, Jan. 2010.

[19] E. Agichtein, C. Castillo, D. Donato, A. Gionis, and G. Mishne, 'Finding High-quality Content in Social Media', in Proceedings of the 2008 International Conference on Web Search and Data Mining, New York, NY, USA, 2008, pp. 183-194.

[20] J. Breslin and S. Decker, 'The Future of Social Networks on the Internet: The Need for Semantics', IEEE Internet Comput., vol. 11, no. 6, pp. 86-90, Nov. 2007.

[21] 'LinkedIn', ProgrammableWeb. [Online]. Available: http://www.programmableweb.com/api/linkedin. [Accessed: 18-May-2015].

[22] 'Twitter Developers', Twitter Developers. [Online]. Available: https://dev.twitter.com/. [Accessed: 25Nov-2015].

[23] 'Mendeley/mendeley-javascript-sdk', GitHub. [Online]. Available: https://github.com/Mendeley/mendeleyjavascript-sdk. [Accessed: 23-Nov-2015].

[24] M. Salman, K. Star, A. Nussbaumer, M. Fuchs, H. Brocks, D. B. Vu, D. Heutelbeck, and M. Hemmje, 'Towards Social Media Platform Integration with an Applied Gaming Ecosystem', presented at the SOTICS 2015, The Fifth International Conference on Social Media Technologies, Communication, and Informatics, 2015, pp. 14-21.

[25] 'Cross-Origin Resource Sharing'. [Online]. Available: http://www.w3.org/TR/cors/. [Accessed: 23-Nov-2015].

[26] D. Gotterbarn, K. Miller, and S. Rogerson, 'Software engineering code of ethics', Commun. ACM, vol. 40, no. 11, pp. 110-118, 1997. 